\title{
Haemophilus influenzae, Penicillinase Positive
}

National Cancer Institute

\section{Source}

National Cancer Institute. Haemophilus influenzae, Penicillinase Positive. NCI Thesaurus.

Code C123480.

Any bacterial species identified as Haemophilus influenzae that produces the enzyme

penicillinase. 